\title{
Développement embryonnaire et clonage chez les mammifères
}

\author{
JP Renard, Y Heyman \\ INRA, biologie du développement, 78352 Jouy-en-Josas, France
}

(Rȩ̣u le 14 septembre 1991; accepté le 15 novembre 1991)

\begin{abstract}
Résumé - Le clonage embryonnaire par transfert d'un noyau dans le cytoplasme d'ovocytes énucléés offre un nouveau champ d'application à la biotechnologie que constitue le transfert d'embryons. Dans l'industrie de l'élevage bovin par exemple, plusieurs séries d'une dizaine de veaux identiques (clones) ont déjà vu le jour. Toutefois moins de $10 \%$ des embryons reconstitués sont capables de se développer à terme après transplantation dans une femelle receveuse. Des progrès sont indispensables si l'on veut que cette technologie puisse être source d'applications, par exemple pour la diffusion de génotypes sélectionnés sur des caractères peu héritables, et la création de nouveaux progrès génétiques. Ces progrès dépendent d'une meilleure compréhension de 2 événements fondamentaux, liés entre eux, qui marquent le début du développement embryonnaire : l'effet du cytoplasme de l'ovocyte sur l'organisation du noyau et la programmation de l'activité des gènes qui est déjà engagée dans le noyau donneur avant son transfert.
\end{abstract}

embryon / clonage / ovocyte / mammifère / gène

Summary - Embryo development and cloning in mammals. Embryonic cloning by transfer of a nucleus to the cytoplasm of enucleated oocytes has opened up new application possibilities in embryo transfer biotechnology. In the bovine industry, for example, several series of about 10 identical calves (clones) have been bom. However, $<10 \%$ of reconstituted embryos are able to develop to term after transplantation to a recipient female. Progress is necessary to be sure that this technique can be developed, for example for the diffusion of genotypes selected on traits of low heritabilities and to make further genetic progress. This progress depends on improved knowledge of 2 linked basic mechanisms which mark the onset of embryonic development : the effect of oocyte cytoplasm on the organization of the nucleus and the gene activity programme already underway in the donor nucleus before its transfer. In mammals the cloned embryo constitutes a new experimental model for analysing the role of factors present in the oocyte cytoplasm. These factors, stocked as messenger RNA and polypeptides during oogenesis, play a determining role in the control of genomic expression during the first segmentation divisions and probably until the first differentiation at the blastocyst stage.

embryo / cloning / oocyte / mammal/gene 


\section{INTRODUCTION}

L'obtention en grand nombre d'individus génétiquement identiques est une pratique couramment utilisée dans les productions végétales. Jusqu'à une date récente, elle n'était pas envisageable pour les productions animales; la situation est en train de changer en particulier en ce qui concerne les mammifères. Cette évolution est due à une meilleure connaissance du début du développement de l'embryon et à la mise au point de nouvelles technologies de micromanipulation.

Ces dernières années la scission de l'embryon au stade préimplantatoire de son développement a commencé à être utilisée au niveau des élevages pour produire des paires de jumeaux notamment chez les bovins. Mais cette approche est limitée; elle ne permet que très rarement d'obtenir des triplés et seulement occasionnellement des quadruplés (Willadsen, 1989). Une autre approche se dessine, elle consiste à réaliser en série la greffe d'un noyau, provenant d'une cellule d'un embryon "donneur", dans le cytoplasme d'un ovule "receveur» dont on a, au préalable, retiré le matériel nucléaire. Les chances de développement à terme des embryons ainsi reconstitués sont aujourd'hui encore faibles. Toutefois cette biotechnologie ouvre la voie à la production de véritables clones, c'est-à-dire d'un ensemble de plusieurs individus génétiquement identiques tous issus d'un même embryon. Ces clones constitueront un nouvel outil pour la diffusion et la création de progrès génétiques.

\section{L'EFFICACITÉ ACTUELLE DU CLONAGE EMBRYONNAIRE}

La technique du transfert nucléaire et son adaptation aux embryons de différentes espèces a fait l'objet récemment de plusieurs revues (Marx, 1988; Willadsen, 1989; Prather et al, 1990; Heyman et al, 1991). Elle consiste d'abord à dissocier les blastomères d'un embryon donneur alors qu'il est encore au début de son développement et possède 8-60 cellules (ce qui est aisément obtenu en les plaçant pendant 15-30 min dans un milieu sans calcium), puis à les fusionner individuellement avec un ovocyte préalablement énuclée. La définition d'une technique originale d'énucléation et de fusion d'embryons de souris par Mc Grath et Solter (1983) a grandement contribué à assurer le renouveau d'une technologie, largement utilisée chez les amphibiens depuis de longues années (Briggs et King, 1952), mais qui n'était jusqu'alors applicable qu'avec beaucoup d'incertitude chez la souris (IIImensee et Hope, 1981). Ainsi, aujourd'hui un expérimentateur confirmé peut, en routine, reconstituer une cinquantaine d'embryons par jour.

Peu d'entre eux toutefois sont capables de se développer à terme. Le tableau I présente les principaux résultats publiés à ce jour chez les espèces domestiques. II montre que les embryons reconstitués sont capables de se diviser et de commencer leur développement. Mais leur mortalité augmente rapidement avec le temps de culture ou après transfert dans une femelle receveuse. On estime aujourd'hui que le rendement global des manipulations, c'està-dire le nombre de jeunes nés par rapport au nombre d'embryons initialement reconstitués est inférieur à 10\%. Chez une espèce comme le lapin, nous avons montré que le taux de développement des embryons "clonés" diminuait rapidement tout au long de la gestation (Heyman et al, 1990), ceci malgré une reprogrammation effective et apparemment normale de l'activité du noyau donneur jusqu'au stade blastocyste. II en résulte qu'à ce jour la taille des clones, c'est-à-dire le nombre d'ani- 
Tableau I.. Développement après transfert de noyaux (données publiées).

\begin{tabular}{|c|c|c|c|c|c|c|}
\hline \multirow[b]{2}{*}{ Espèce } & \multicolumn{3}{|c|}{ In vitro } & \multicolumn{3}{|c|}{ in vivo } \\
\hline & $\begin{array}{l}\text { Embryon } \\
\text { donneur }\end{array}$ & $\begin{array}{c}\text { Morula et } \\
\text { blastocystes }\end{array}$ & $(\%)$ & $\begin{array}{c}n b \text { de } \\
\text { jeunes nés }\end{array}$ & $\begin{array}{c}\text { clones } \\
\mathrm{n}=\end{array}$ & Références \\
\hline Vache & $\begin{array}{r}4-16 \text { cell } \\
16-64 \text { cell } \\
30-40 \text { cell } \\
8-64 \text { cell }\end{array}$ & $\begin{array}{r}23 / 185 \\
280 / 1123 \\
28 / 108\end{array}$ & $\begin{array}{l}12 \\
25 \\
32\end{array}$ & $\begin{array}{r}2 \\
92 \\
101\end{array}$ & $\begin{array}{l}8 \\
4\end{array}$ & $\begin{array}{l}\text { Prather ot al (1987) } \\
\text { Bondioli et al (1990) } \\
\text { Simms et al (1991) } \\
\text { Willadsen ot al (1991) }\end{array}$ \\
\hline Brebis & $\begin{array}{r}8-16 \text { cell } \\
16 \text { cell } \\
\text { Blastocyste (BE) }\end{array}$ & $\begin{array}{r}46 / 105 \\
22 / 95 \\
9 / 22\end{array}$ & $\begin{array}{l}43 \\
23 \\
40\end{array}$ & $\begin{array}{l}3 \\
2 \\
1\end{array}$ & 2 & $\begin{array}{l}\text { Willadsen (1986) } \\
\text { Smith et Wilmut (1989) } \\
\text { Smith et Wilmut (1989) }\end{array}$ \\
\hline Lapin & $\begin{array}{r}8 \text { cell } \\
32 \text { cell congelé } \\
32 \text { cell frais } \\
\text { Blastocyste (BE) }\end{array}$ & $\begin{array}{r}35 / 108 \\
32 / 72 \\
13 / 64\end{array}$ & $\begin{array}{l}32 \\
44 \\
20\end{array}$ & $\begin{array}{r}6 \\
8 / 207 \\
10 / 139\end{array}$ & $\begin{array}{l}6 \\
6\end{array}$ & $\begin{array}{l}\text { Stice et Robl (1988) } \\
\text { Heyman et al (1990) } \\
\text { Collas et Robl (1991) } \\
\text { Collas et Robl (1991) }\end{array}$ \\
\hline Porc & 4 cell & $7 / 83$ & 8 & $1 / 88$ & & Prather et al (1989) \\
\hline Chèvre & 8-32 cell & & & $5 / 24$ & 2 & Yong et al (1991) \\
\hline
\end{tabular}

maux issus du même embryon reste faible. La dizaine constitue un record mais ces clones existent et témoignent de la faisabilité de cette biotechnologie.

\section{DES PROGRĖS PRÉVISIBLES}

L'artifice mis en cuvre dans le transfert nucléaire repose sur l'établissement de relations fonctionnelles entre le cytoplasme de l'ovocyte receveur et le noyau du blastomère donneur.

Le cytoplasme de l'ovocyte est pleinement capable de réorganiser un noyau étranger et de le rendre compétent pour participer au développement normal. C'est en effet cette situation qui prévaut au cours de la fécondation. La chromatine du noyau du spermatozoïde est soumise à une décondensation rapide qui se produit en même temps que l'achèvement de la méĩose de l'ovocyte (Adenot et al, 1990). Cette modification qui permet le remplacement progressif des protamines par les histones (Nonchev et Tsanev, 1990) est suivie par une phase de décondensation rapide qui correspond à la formation d'une nouvelle enveloppe nucléaire constituée à partir d'éléments stockés dans l'ovocyte. Nous avons montré que l'on pouvait suivre ces modifications de l'état de la chromatine du noyau in situ sur les œufs vivants en utilisant une technologie nouvelle, la microscopie de fluorescence basse énergie (Debey et al, 1989). On ne sait pas encore comment le cytoplasme de l'ovocyte participe à la réorganisation de la membrane nucléaire, mais on sait par contre qu'après transfert de noyau, l'acquisition d'une nouvelle structure membranaire est 
une condition nécessaire à la formation d'un noyau diploïde fonctionnel (Szöllösi et al, 1988). Le remodelage du noyau conditionne donc son aptitude à se diviser et à permettre la réalisation à nouveau des premières étapes du développement. Le tableau II montre que ces évènements se produisent dès les premières $h$ qui suivent leur transfert dans le cytoplasme de l'ovocyte.

Le noyau donneur au moment de son prélèvement a déjà subi plusieurs cycles de réplication et est déjà le plus souvent capable d'une activité transcriptionnelle. Chez les mammifères en effet, la mise en route du génome embryonnaire se produit au cours des premières divisions de l'œuf. Chez la souris, elle s'effectue en 2 phases au cours du stade 2 cellules (Bensaude et al, 1983; Conover et al, 1991). La première est caractérisée par la néosynthèse d'un groupe de protéines qui correspond aux protéines dites de choc thermique. La seconde se produit quelques $h$ après ( $8 \mathrm{~h}$ environ) avec la synthèse de plusieurs nouvelles protéines. Chez des espèces comme la lapine (données non publiées) ou la vache (Barnes et Eyestone, 1990), la mise en activité du génome semble être plus progressive que chez la souris et faire intervenir plusieurs autres synthèses protéiques encore peu caractérisées. C'est peut-être cette différence qui fait que, malgré de nombreuses expériences, le clonage par transfert de noyau chez la souris se traduit par des taux de développement encore plus faibles que ceux obtenus chez les espèces domestiques (Kono et al, 1991).

Au cours du développement normal, la synthèse de quelques protéines nouvelles peut être décelée à partir de la première division de segmentation (stade 2) chez la lapine, ou la deuxième (stade 4) chez la vache, mais le profil de synthèse n'est modifié de façon marquée qu'à partir du stade 8-16 cellules. L'initiation de cette première phase de l'activité transcriptionnelle dépend étroitement du temps écoulé après l'activation de l'ovocyte. Elle met en jeu une succession de protéines kinases qui contrôlent probablement l'accessibilité de facteurs de transcription et l'activité de la polymérase 2. La deuxième phase de l'activation transcriptionnelle du génome ne peut, contrairement à la première, se produire que si la replication de I'ADN a bien eu lieu au cours du stade précédent (Poueymirou et Schultz, 1989). Elle coïncide chez la souris avec des exigences métaboliques nouvelles de l'embryon.

La cinétique de ces évènements moléculaires après transfert de noyau n'est pas encore connue. II en est de même en ce qui concerne l'implication des protéines qui sont synthétisées pendant la période préimplantatoire du développement. Les quelques synthèses qui ont pu être caractérisées (principalement chez la souris) après fécondation, révèlent la présence de phosphoprotéines dont l'activité est liée au cycle cellulaire, de lamines qui participent à la formation de la membrane nucléaire, de catherines impliquées dans les communications entre cellules et d'oncogènes qui participent à la régulation de l'activité des gènes et des divisions de segmentation (Jackson, 1989).

Les noyaux de la masse cellulaire interne sont encore capables d'assurer un développement à terme (Smith et Wilmut, 1989) alors que ce n'est pas le cas pour les noyaux du trophoblaste. Le noyau embryonnaire conserve donc une certaine plasticité fonctionnelle pendant les premiers jours qui suivent la fécondation. Chez le lapin, nos résultats montrent que si l'on prend en compte la cinétique des événements qui réorganisent le noyau après son transfert, on peut considérablement augmenter le taux de développement in vitro (tableau II). Des voies semblent donc pouvoir être ouvertes dans les enchevêtrements complexes sous lesquels 
Tableau II . Développement in vitro des embryons "clonés" de lapin selon l'état de leur noyau 2 h après fusion

\begin{tabular}{lcccc}
\hline & & \multicolumn{3}{c}{$\begin{array}{c}\text { Aspect de la chromatine } \\
\text { 2h après transfert nucléaire a }\end{array}$} \\
\cline { 3 - 5 } $\begin{array}{l}\text { Nombre } \\
\text { d'embryons }\end{array}$ & Total & remodelée b & non modifiée & condensée \\
\hline Observés & 49 & 29 & 9 & 11 \\
$\begin{array}{l}\text { Activés cobservés } \\
\text { (\%) }\end{array}$ & $30 / 49$ & $27 / 29$ & $2 / 9$ & $1 / 11$ \\
$\begin{array}{l}\text { blastocystes/observés } \\
(\%)\end{array}$ & $(61 \%)$ & $(93 \%)$ & $(22 \%)$ & $(9 \%)$ \\
& $12 / 38$ & $12 / 22$ & $0 / 9$ & $0 / 11$ \\
\hline
\end{tabular}

a Les embryons sont observés en microscopie de fluorescence basse énergie après marquage spécifique du noyau par un fluorochrome (Adenot et al, 1990).

b Gonflement marqué de la chromatine : volume du noyau multiplié par 5.

c Embryons au stade 2 cellules $20 \mathrm{~h}$ après fusion.

se manifestent aujourd'hui les relations entre le noyau embryonnaire et le cytoplasme du début de la vie de l'embryon.

\section{QUELLES APPLICATIONS POUR L'ÉLEVAGE?}

L'élevage français connait aujourd'hui une évolution rapide marquée notamment par l'établissement de nouvelles relations avec le secteur en pleine expansion des agroindustries. Celles-ci dégagent annuellement un excédent de 30 milliards de francs et l'élevage doit diversifier, voire redéfinir ses productions pour maintenir sa place de secteur exportateur. La sélection est un outil de cette adaptation et les connaissances nouvelles acquises sur le génome avec l'établissement progressif des cartes génétiques par espèce seront un atout pour l'élevage. Mais l'adaptation doit être rapide : un des freins à cette voie de progrès est la faible efficacité reproductrice et l'intervalle de génération élevé de la plu- part des espèces. II peut être levé grâce à l'embryon. Les recherches sur la biologie du développement embryonnaire ont rendu possible, ces dernières années, des interventions en routine sur l'embryon au début du développement et donné à la conduite de la reproduction une place nouvelle dans la sélection des espèces animales. Notre pays, en ce qui concerne les bovins, est en tête des pays européens pour l'utilisation de la transplantation embryonnaire, les échanges d'embryons congelés et le sexage embryonnaire commencent à être utilisés par plusieurs coopératives d'élevage. Si la technologie du clonage progresse, nul doute que c'est d'abord dans l'espèce bovine qu'elle commencera à être utilisée.

Des clones, de taille réduite à quelques individus, peuvent contribuer à la création de progrès génétique. On a calculé qu'en production laitière, un clone de 5 vaches indexées sur leur propre production permettrait de connaître la valeur génétique d'un taureau avec la même précision que 
celle obtenue à partir d'une indexation sur 25 de ses filles (Colleau, 1991). En race à viande, l'existence de quelques clones permettrait de mieux évaluer les aptitudes maternelles.

Avec des clones de taille plus importante (une dizaine d'individus) une stratégie de production d'animaux très standardisée - sous label - pourrait être mise en place avec des répercussions rapides sur la diffusion du progrès génétique. Seront concernés les animaux exceptionnels, avec la perspective de réduire le coût de l'embryon transplanté (Lapin, 1991) et comme conséquence, une intensification de l'utilisation de la transplantation embryonnaire dans un programme de sélection. Mais le clonage permettra aussi, sur un plan collectif, de mieux articuler diffusion et création de progrès génétique, notamment si on peut lui associer la congélation pour utiliser les meilleurs clones et assurer alors à nouveau une diffusion. Dans ces conditions une augmentation de $40 \%$ de l'efficacité génétique d'un programme peut être envisagée (Colleau, 1990).

\section{CONCLUSION}

À plus long terme, l'obtention régulière de clones de plusieurs dizaines d'individus rendra plus aiguē la difficile question du maintien d'un polymorphisme génétique pour les espèces d'intérêt zootechnique. Mais cette question se posait déjà depuis de longues années avant que le clonage n'ait vu le jour. Certes le recyclage des embryons clonés est possible et quelques veaux issus d'embryons clonés de troisième génération sont déjà nés (Bondioli et al, 1990). Raison de plus pour souligner que c'est une gestion stricte des ressources génétiques qui seule permettra de préserver la diversité génétique des animaux de ferme.

\section{RÉFÉRENCES}

Adenot PG, Szollosi M, Geze M, Renard JP, Debey $P(1990)$ Dynamics of paternal chromatin changes in live one cell mouse embryo after natural fertilization. Mol Reprod Dev 28, 23-34

Barnes FL, Eyestone WH (1990) Early cleavage and the maternal zygotic transition in bovine embryos. Theriogenology 33, 141-152

Bensaude $O$, Babinet $C$, Morange $M$, Jacob $F$ (1983) Heat shock proteins, first major products of zygotic gene activity in mouse embryo. Nature 305, 331-333

Bondioli KR, Westhusin ME, Looney CR (1990) Production of identical bovine offspring by nuclear transfer. Theriogenology 33, 165-174

Briggs R, King TJ (1952) Transplantation of living cell nuclei from blastula cells into enucleated frog's eggs. Proc Natl Acad Sci USA 38, 455-463

Collas PH, Robl JM (1991) Development of rabbit nuclear transplant embryos from morula and blastocyst stage donor nuclei. Theriogenology 35, 190 (abstr)

Colleau JJ (1990) Quelles conséquences attendre des nouvelles techniques de reproduction en matière d'amélioration génétique ? $1^{r e}$ Journ UNLG, Paris, 21 nov 1990

Colleau JJ (1991) Combined use of embryos sexing and cloning within closed mixed MOETs for selection on dairy cattle. 42nd annu meet EAAP. Berlin, 8-12 sept 1991, session 4

Conover JC, Temeles GL, Zimmermann JW, Burke B, Schultz RM (1991) Stage-specific expression of a family of proteins that are major products of zygotic gene activation in the mouse embryo. Dev Biol 144, 392-404

Debey P, Renard JP, Coppey-Moisan M, Monnot I, Geze M (1989) Dynamics of chromatin changes in live one-cell mouse embryos : a continuous follow-up by fluorescence microscopy. Exp Cell Res 183, 413-433

Heyman Y, Chesné P, Renard JP (1990) Reprogrammation complète de noyaux embryonnaires congelés, après transfert nucléaire chez le lapin. CR Seance Acad Sci Paris Sér III 311, 321-326

Heyman $Y$, Chesné $P$, Renard JP (1991) Transplantation de noyaux et obtention de clones 
chez les mammifères domestiques. Rec Méd Vét 167 (3/4) 315-322

IIImensee K, Hoppe PC (1981) Nuclear transplantation in Mus musculus. Developmental potential of nuclei from preimplantation embryos. Cell 23, 9-18

Jackson IJ (1989) Genes and embryos (Glover and Hames, eds) IRL Press, 165-221

Kono $T$, Kwon OY, Ogawa $M$, Nakahara $T$ (1991) Development of mouse oocytes receiving embryonic nuclei and thymocytes. Theriogenology 35 (1) 227 (abstract)

Lapin (1991) Granada Biosciences, leader dans les biotechnologies. Typex 17, fev 1991

Marx JL (1988) Cloning sheep and cattle embryos. Science 239, 463-464

Mc Grath J, Solter D (1983) Nuclear transplantation in the mouse embryo by microsurgery and cell fusion. Science 220, 1300-1302

Nonchev S, Tsanev R (1990) Protamine-histone replacement and DNA replication in the male mouse pronucleus. Mol Reprod Dev 25, 7276

Poueymirou WT, Schultz RM (1989) Regulation of mouse preimplantation development: inhibition of synthesis of proteins in the two cell embryo that require transcription by inhibition of CAMP dependent protein kinase. Dev Biol 133, 588-589

Prather RS, Barnes FL, Sims MM, Robl JM, Eyestone WH, First NL (1987) Nuclear transplantation in the bovine embryo : assessment of donor nuclei and recipient oocyte. Biol Reprod 37, 859-866
Prather RS, First NL (1990) Nuclear transfer in mammalian embryos. Int Rev Cytol 120 , 169-190

Prather RS, Sims MM, First NL (1989) Nuclear transplantation in early pig embryos. Biol Reprod 41, 414-418

Sims MM, Rosenkrans CF, First NL (1991) Development in vitro of bovine embryos derived from nuclear transfer. Theriogenology 35, 272 (abstr)

Smith LC, Wilmut I (1989) Influence of nuclear and cytoplasmic activity on the development in vivo of sheep embryos after nuclear transplantation. Biol Reprod 40, 1027-1035

Stice SL, Robl JM (1988) Nuclear reprogramming in nuclear transplant rabbit embryos. Biol Reprod 39, 657-664

Szöllösi D, Czolowska A, Szollosi MS, Tarkowski AK (1988) Remodeling of mouse thymocyte nuclei depends on the time of their transfer into activated, homologous oocytes. J Cell Sci 91, 603-613

Willadsen SM (1986) Nuclear transplantation in sheep embryos. Nature 320, 63-65

Willadsen SM (1989) Cloning of sheep and cow embryos. Genome 31, 956-962

Willadsen SM, Janzen RE, McAlister RJ, Shea B, Hamilton G, McDermand D (1991) The viability of late morulae and blastocysts produced by nuclear transplantation in cattle. Theriogenology 35, 161-170

Yong Z, Jianchen W, Jufen Q, Zhiming $H$ (1991) Nuclear transplantation in goats. Theriogenology 35, 299 (abstr) 